$4.75 \mathrm{~cm} / \mathrm{sec}$. The recording medium consists of submicroscopic iron particles. Because of the high magnetic remanence of the material. thickness of the magnetic layer can be much less than conventional oxide tapes. This reduced thickness does not reduce low-frequency output, yet allows much higher output at high frequencies. Noise level remains constant, so $\mathrm{S} / \mathrm{N}$ ratio improves, according to the developers.

\section{Midget Lamp}

A midget 13-W tungsten-halogen lamp has been developed by the General Electric Miniature Lamp Department for uses as microscope illuminators, fiber optic sources, and other instruments. The lamp operates on $6.3 \mathrm{~V} \mathrm{dc}$, and light output is $15 \mathrm{cp}$. The lamp maintains a nearly constant level of brightness over its 75 -h average rated lifespan. Price is $\$ 6.43$ per unit.

\section{MODCOMP IV}

Modular Computer Systems recently have introduced the MODCOMP IV, a dual word processor with 32-bit parallel internal organization. 64-bit floating-point hardware, 240 general-purpose registers, and a 32K-byte core memory expandable to $512 \mathrm{~K}$ bytes. Prices for the $32 \mathrm{~K}$-byte machines start at $\$ 18,500$. Cycle time is $640 \mathrm{nsec}$ in both core and solid state; fast context switching is done with 16 sets of 15 general-purpose registers in each set and 1,024 address mapping registers.

\section{Tiny CCD TV Camera}

Fairchild Camera and Instrument Corp., of Mountain View. Calif.. has announced the MV 100 , the first commercial charge-coupled-device television camera. The unit is palm sized and contains a 100 by 100 array of photosensitive CCDs and is claimed to be the first of a series that will eventually replace vidicon TV cameras in many applications. The unit has a sensitivity of $0.1 \mathrm{fc}$, so the CCD camera can operate under a broad range of lighting conditions. The camera weighs $60 z$, measures $3.5 \times 1.5 \times 2.25$ in., and consumes only $1 \mathrm{~W}$ of power. Disadvantages of the MV 100 include a lower resolution than that of a conventional TV camera (only 16 lines $/ \mathrm{mm}$ ), and it cannot be used on a conventional TV monitor. The vertical synch frequency must be changed to accommodate the CCD camera, but this can be done by merely changing one resistor. At this writing, the price has not been announed.

\section{New German Minicomputer}

Krupp Atlas-Elektronik has introduced a new minicomputer, the EPR 1100. Modules assembled on PC boards are drawer mounted. the basic drawer containing the CPU. Working memory utilizes either RAM or ROM/PROM with 800-nsec RAM cycle time. The basic $4 \mathrm{~K} 16$-bit word memory is expandable to $64 \mathrm{~K}$ with $4 \mathrm{~K}$ RAM modules. The OPS 1100 software system contains programs for $1 / 0$, process, interrupt and time control. as well as loading and routines. and programs for connected peripherals. An assembler (ASS 1100) is available for setting up programs.

\section{Micro-Adjust Cams}

Gemco is marketing new micro-adjust rotating cam limit switches featuring: precision cam adjustment at any angular position of the cam shaft, speeds from 0 to $500 \mathrm{rpm}$ in either direction, no special cam requirement for any contact setting from 4 to $356 \mathrm{deg}$, and no tools required to adjust cam settings.

A descriptive catalog may be obtained by writing Gemco Electric Co.. Clawson, Mich. 48017.

Psychological Instruments

A new catalog with a price list effective September 1973 is available from Psychological Instruments Co., P.O. Box 6113, Richmond, Va. 23222.

\section{Notes}

\section{A measure of spontaneous alternation}

\author{
SUSAN KARP MANNING* \\ Hunter College of the City University of New York \\ New. York, New' York 10021
}

Spontaneous alternation, the above-chance probability of differing successive choices in a two-alternative situation, has been studied in both rat (see Dember \& Fowler, 1958, for a review) and human Ss (e.g. Manning \& Artman. 1973).

However, although this paradigm is seemingly simple, the measurement of the spontaneous alternation tendency has presented many difficulties. Most of these have related to incorrect assessment of the expected chance level of alternation (EA). These problems appear to have been solved (Douglas, 1964, 1966: Manning. 1973).

Although this is the case. none of the previously devised methods of analyses have provided a fully satisfactory measure. These methods either do not lead to descriptive scores that are comparable from experiment to experiment: do not express observed alternation $(\mathrm{OA})$ as a proportion of possible above-chance alternation; and/or do not lead to measures that have a value of 1.0 when alternation has occurred on all trials. A measure is developed here which accomplishes these goals.

This measure. the adjusted alternation ratio (AAR). appears below as Eq. 1. Define $\mathrm{N}$ as the number of alternation trials given. Thus. NEA is the actual number of expected alternations:

\footnotetext{
* Please Address reprint requests to Susan Karp Manning. Department of Psychologr. Hunter College. 695 Park Avenue. New York. New York 10021.
} 


$$
A A R=\frac{O A-N E A}{N-N E A}
$$

An example of the use of Eq. 1 will clarify the situation. Define the S's two choices as $M$ and $O$ and the probability of choice of $M$ as $m$ and of $O$ as $(1-m)$. Dember and Fowler's (1958) expression for EA for a given $S$ in the free-trial paradigm may be written as Eq. 2:

$$
E A=2 m(1-m)
$$

An unbiased estimate of Eq. 2 may be obtained by counting the number of $M$ choices $(k)$ on independent trials and dividing by the total number of choice opportunities (N) (Manning, 1973). This expression is represented as Eq. 3:

$$
E A=2(k / N)(1-k / N)(N / N-1) \text {. }
$$

As is evident from these equations, EA depends on the deviation of $\mathrm{m}$ from 0.5 . As this deviation or preference for one alternative increases, EA gets smaller. Thus, for example, NEA values for Ss choosing $M$ on 7 and 9 of 10 choices are 4.70 and 2.0, respectively. If each $S$ is given 10 opportunities to alternate, the difference between the maximum alternation possible and NEA differs for the two Ss and mere deviation scores do not reflect true relative alternation differences. Further, if different numbers of trials are given, maximum deviations differ and deviation scores which are actually larger may represent smaller true alternation tendencies.

Thus, a score which expresses the difference between $O A$ and NEA as a proportion of the difference between $\mathbf{N}$ and NEA provides a measure which makes the amount of alternation comparable for different preferences and different numbers of trials. In the example above, AAR $=.43$ and .88 , respectively. These numbers represent the proportion of above-chance alternation present as compared to the total amount possible. Examination of Eq. 1 shows AAR to equal 1.0 if the $S$ always alternates $(\mathrm{OA}=\mathrm{N})$.

This measure may be applied to the forced-trial paradigm (where $S$ receives a forced first choice and a free second choice) by using the appropriate equation to obtain EA (Manning, 1971).

In the above form, AAR can only be used if $O A \geqslant N E A$. If this condition is not met, a negative AAR (NAAR) or an adjusted repetition ratio may be obtained from Eq. 4:

$$
\text { NAAR }=\frac{O A-N E A}{N E A}
$$

This measure has the same properties as AAR and should be applied to data when OA $<$ NEA. Further, it may be used to test for the presence of a significant repetition tendency.
One additional point may be made. This measure, although applied here to the spontaneous alternation paradigm, is mathematically identical with the ARC (adjusted ratio of clustering) measure developed by Roenker, Thompson, \& Brown (1971) and frequently used by others to measure clustering of word categories in the free-recall learning situation. It is undoubtedly the case that there are other situations commonly studied in the psychological literature where adaptations of this equation would be useful.

\section{REFERENCES}

Dember, W. N., F Fowler, H. F. Spontaneous alternation behavior. Psychological Bulletin, 1958, 55, 412-428.

Douglas, R. J. Spontaneous alternation cues. Unpublished doctoral dissertation, University of Michigan, 1964 .

Douglas, R. J. Cues for spontaneous alternation. Joumal of Comparative \& Physiological Psychology, 1966, 62, 171-183.

Manning, S. K. A method of assessing the chance level of alternation in the forced-trial paradigm. American Journal of Psychology, 1971, 84, 421-424.

Manning, $S$. $K$. A design comparing free- and forced-trial alternation in the rat at two interchoice intervals. Behavior Research Methods \& Instrumentation, 1973, 5, 263-265.

Manning, S. K., \& Artman, J. A. New procedure to test some factors in human spontaneous altexnation. Journal of Experimental Psychology, 1973, 97, 274-277.

Roenker, D. L., Thompson, C. P., \& Brown, S. C. Comparison of measures for the estimation of clustering in free recall. Psychological Bulletin, 1971, 76, 45-48.

(Received for publication July 3, 1973; accepted August 8, 1973.)

\section{Vibration isolation for small aquaria*}

\author{
RICHARD R. FAY \\ Laboratory of Sensory Sciences \\ University of Hawaii, Honolulu, Hawaii 96822
}

and

\section{ARTHUR N. POPPER}

Department of Zoology and Laboratory of Sensory Sciences University of Hawaii, Honolulu, Hawaii 96822

Behavioral experiments with aquatic animals frequently require that the water tank be isolated from substrate vibrations. Unfortunately, low-frequency noise is transmitted quite efficiently into an unisolated water tank, and although everyone recognizes the need for some isolation, the methods used are usually chosen on the basis of intuition and on the materials handily or cheaply available.

In this paper, we describe and compare the vibration isolation properties of two common laboratory materials that may be suitable as isolation materials for small tanks of water. The materials were a 2-in.-thick piece of new polyurethane foam (Serofoam brand, Sears Roebuck) and two sheets of layered plastic materials with embedded air bubbles commonly received as packing material (referred to commercially as Air Caps).

Testing was as follows. Each of the materials was

*This work was supported by Public Health Service Grants NS-09374 and NS-06890 from the National Institute of Neurological Diseases and Stroke. 